\title{
APPLICATION OF MULTIPLE-SCATTERING POLARIZATION LIDAR FOR THE EVALUATION OF SPACE-BORNE LIDAR ALGORITHMS
}

\author{
Hajime Okamoto $^{1 *}$, Kaori Sato ${ }^{1}$, Shuichiro Katagiri ${ }^{1}$, Masahiro Fujikawa ${ }^{1}$, Tomoaki Nishizawa ${ }^{2}$, \\ Nobuo Sugimoto ${ }^{2}$, Yoshitaka $\mathrm{Jin}^{2}$, Atsushi Shimizu ${ }^{2}$, Hiroshi Ishimoto ${ }^{3}$ \\ ${ }^{1}$ Research Institute for Applied Mechanics: Kyushu University, Kasuga, Kasuga park 6-1, 816-8580, \\ JAPAN, *Email: okamoto@riam.kyushu-u.ac.jp \\ ${ }^{2}$ National Institute for Environmental Studies, Tsukuba, Ibaraki 305-8506, JAPAN \\ ${ }^{3}$ Meteorological Research Institute: Japan Meteorological Agency, Tsukuba Ibaraki 305-0052, JAPAN
}

\begin{abstract}
Multi-field of view multiple-scattering polarization lidar (MFMSPL) has been developed to measure enhanced backscattering and depolarization ratio from optically thick clouds. Collocated cloud radar observations and Monte Carlo simulations supported the system performance such as cloud detectability and values of depolarization ratio. The system is used to evaluate/improve algorithms for space-borne lidar such as CALIOP on CALIPSO and ATLID on EarthCARE.
\end{abstract}

\section{INTRODUCTION}

Multiple scattering effects in lidar signals have been known to be important to analyze cloud phase and microphysical properties [1]. When optical thickness becomes large, single scattering approximation is not valid and non-zero depolarization ratio appears even for spherical particles. This can be explained as follows; number of backscattered photons affected by multiple scattering events increases as optical thickness increase when other factors are the same and multiple scattering process produces non-zero depolarization ratio. These phenomena have been frequently observed for space-borne lidar observations. Observed depolarization ratio often well exceeded $30 \%$ when water clouds were observed as for the case of CALIPSO lidar [2].

Since ice particles often produces large depolarization ratio comparable to that of water clouds in case of space borne lidar observations, there is a fundamental difficulty to use depolarization ratio alone to discriminate cloud phase. To overcome the above situation, it was shown that cloud particle phase could be determined by the combined use of depolarization ratio and the ratio of the attenuated backscattering coefficients for two vertically consecutive layers [3].

We introduced a new ground-based lidar system, Multiple-Field of view Multiple Scattering Polarization Lidar (MFMSPL) that can detect backscattering coefficients and depolarization ratios similar to those observed by CALIPSO lidar [4]. The collocated measurements by $95 \mathrm{GHz}$ cloud radar and the MFMSPL showed good agreement in the detection of cloud top boundaries and Monte Carlo simulations verified the performance of the system and accuracy of the observables.

The main objective of the study is to develop and evaluate space-borne lidar algorithms such as cloud mask scheme and cloud particle type algorithms for the analysis of CALIPSO lidar and high spectral resolution lidar, ATLID, on the EarthCARE satellite mission (JAXA-ESA)[5]. The data can be also used to study radiative impacts of clouds and aerosol-cloud interactions.

\section{DESCRIPTION OF THE MFMSPL}

The MFMSPL has been originally built with eight channels (four parallel and four perpendicular with detectors) and was recently extended to 10channel system. Five parallel- and perpendicular channel telescopes are inclined with different angles from the vertical direction along a line. $\mathrm{CH} 1$, $\mathrm{CH} 3, \mathrm{CH} 5, \mathrm{CH} 7$ and $\mathrm{CH} 9$ detect parallel signals and the tilt angles are $0,10,20,30$, and $40 \mathrm{mrad}$, respectively. $\mathrm{CH} 2, \mathrm{CH} 4, \mathrm{CH} 6, \mathrm{CH} 8$ and $\mathrm{CH} 10$ channels detect perpendicular signals and have tilt angles of $0,10,20,30$, and $40 \mathrm{mrad}$, respectively. FOV of each telescope is $10 \mathrm{mrad}$. N; YAG laser is used and second harmonic wavelength of $532 \mathrm{~nm}$ is detected. The temporal and vertical resolutions of 
the data are $10 \mathrm{~s}$ and $6 \mathrm{~m}$, respectively. The original data are averaged for $5 \mathrm{~min}$ and $48 \mathrm{~m}$ in this study.

\section{CLOUD MASK AND CLOUD PARTICLE TYPE FOR MFMSPL}

\subsection{Cloud detection}

We first evaluated the cloud mask algorithm for CALIPSO lidar, KU-mask [6], by using the MFMSPL data. The KU-mask was originally developed based on the cloud mask algorithm for ship-based Mie-type lidar with small FOV[7][8]. It turned out that the KU mask scheme tended to underestimate cloud top regions when water clouds existed.

We developed new cloud mask algorithm that can be applicable for all of the channels of the MFMSPL. The new cloud mask scheme was designed to properly treat the signals from the deeper part of the clouds for off-beam channels where signals became small above cloud bottom regions. The cloud mask result is shown for the total attenuated backscattering coefficient $\left(\beta_{\text {att,tot }}\right)$ estimated by $\beta_{\text {att }}$ for $\mathrm{CH} 5$ and $\beta_{\text {att }}$ for $\mathrm{CH} 6$ (Figure $1)$.

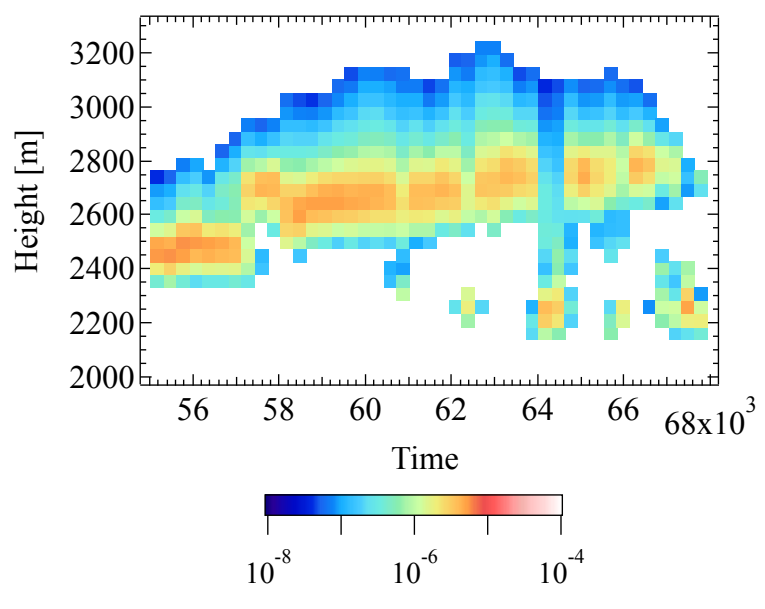

Figure 1 Time-height plot for the total attenuated backscattering coefficient $\beta_{\text {att,tot }}$ for $\mathrm{CH} 5$ and $\mathrm{CH} 6$ of the MFMSPL.

The results for the off-beam channels such as $\mathrm{CH} 3+\mathrm{CH} 4, \mathrm{CH} 5+\mathrm{CH} 6$ and $\mathrm{CH} 7+\mathrm{CH} 8$, showed higher cloud top compared with the on-beam channel $(\mathrm{CH} 1+\mathrm{CH} 2)$. The algorithm has also a function to identify the fully attenuated pixels.

Then we improved the cloud mask algorithm for space borne lidar on the basis of the algorithms for the MFMSPL. The cloud detection in the deeper bottom part of the clouds was improved by the new cloud mask.

\subsection{Analysis of water cloud signatures}

Algorithm for cloud particle type (KU-type) that can be applied to CALIPSO lidar, required depolarization ratio and ratio of attenuated backscattering coefficients for the two vertically consecutive layers [3]. The logarithmic expression of the ratio X' was used in the algorithm.

$$
X^{\prime}\left(R_{i}\right)=\log _{10}\left[\frac{\beta_{a t t}\left(R_{i}\right)}{\beta_{a t t}\left(R_{i+1}\right)}\right]
$$

, where $R_{i}$ denotes the distance of the center of the cloud layer $\mathrm{i}$ viewed from the satellite. and $\mathrm{R}_{\mathrm{i}+1}$ denotes the next layer $i+1$. $\beta_{\text {att }}$ denotes the attenuated backscattering coefficient at layer i. X' was introduced as a proxy of the layer extinction. The two-dimensional diagram on $X^{\prime}$ and depolarization ratio was created based on the occurrence frequencies of water clouds, randomly oriented ice and horizontally oriented ice particles from CALIPSO lidar signals.

Similarly, $X^{\prime}$ was estimated using the observed values by the MFMSPL observations with modifications, where $R_{i}$ is taken to be the cloud height and $\mathrm{R}_{\mathrm{i}+1}$ corresponded to the next upper layer. Here we examined the depolarization ratio and X' for water clouds observed by the MFMSPL. Time height plot for depolarization ratio and $X$ ' was shown in Figures $2 \mathrm{a}$ and $2 \mathrm{~b}$, respectively.

Analyses of vertical distribution of the depolarization ratio of water clouds showed the values in general monotonically increased from cloud bottom to cloud top. Maximum of depolarization ratio often exceeded $80 \%$ near the cloud top regions. On the other hand, $X$ ' first increased up to the middle part of the cloud as height increased and turned to slightly decrease or became nearly constant toward cloud top. Large negative values (-1) of $X^{\prime}$ were found near cloud bottom regions where $\beta_{\text {att,tot }}$ increased to some of cloud middle parts. 


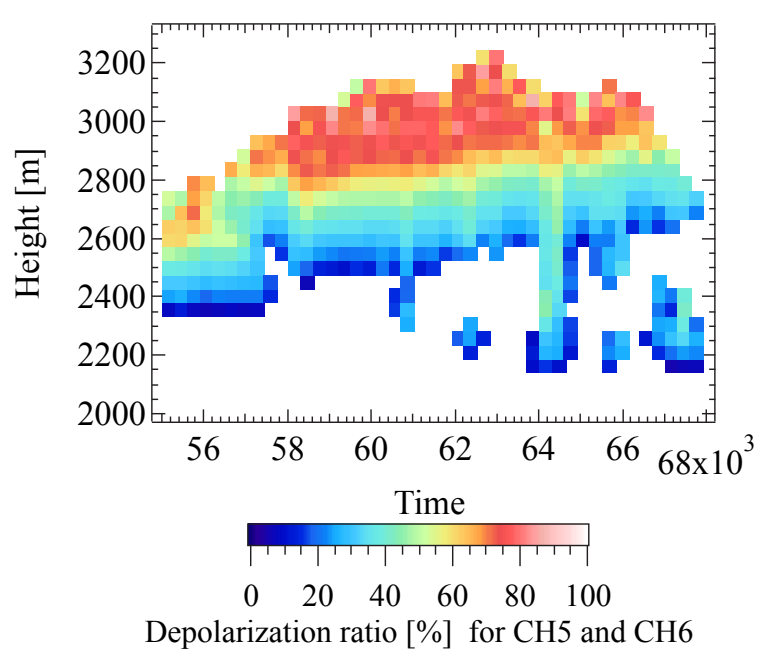

(a)

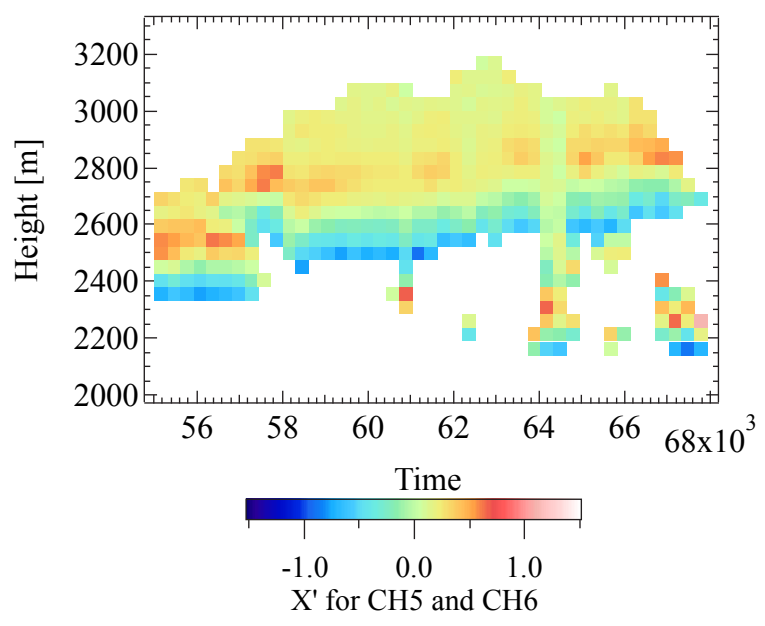

(b)

Figure 2(a) Time-height plot for the depolarization ratio estimated by $\beta_{\text {att }}$ for $\mathrm{CH} 5$ and that for $\mathrm{CH} 6$ for the same clouds in Figure 1. (b) Same as (a) but for X'.

Next, relationship between depolarization ratio and $X^{\prime}$ was examined (Figure 3). There are several features found in the relationship. For depolarization ratio smaller than about $40 \%$, as X' increased, depolarization increased for the water clouds. Compared with the diagram used in the KU-type for CALIPSO lidar, the value of depolarization ratio in this analysis were somewhat larger for the same $X^{\prime}$. This might be explained as follows; the CALIPSO backscattered signals were results of the integration of signals from both of inner and outer parts. Depolarization ratio from outer part is expected to be larger than that from inner part since signals from outer part are more affected by the multiple scattering contributions compared with the inner parts. Actual observations revealed, i.e., observed depolarization ratio estimated by $\mathrm{CH} 5$ and $\mathrm{CH} 6$ is larger than that by $\mathrm{CH} 1$ and $\mathrm{CH} 2$ as expected.

For the depolarization ratio $>40 \%$, $\mathrm{X}^{\prime}$ decreased as depolarization ratio increased. These data correspond to the upper cloud layers above about $2800 \mathrm{~m}$. These large depolarization ratio with small $\mathrm{X}^{\prime}$ also was found when ice particles appeared so that there is a difficulty to discriminate water from ice particles in these parameter space. Thus it might be necessary to introduce additional discrimination scheme as follows. The cloud phase for the upper regions is considered to be water when the cloud phase of the lower cloud layer connected to the upper layer of interest is identified as water.

Similar modification in the cloud particle type algorithm might be needed for the analysis of cloud phase retrieved from space borne lidar observations.

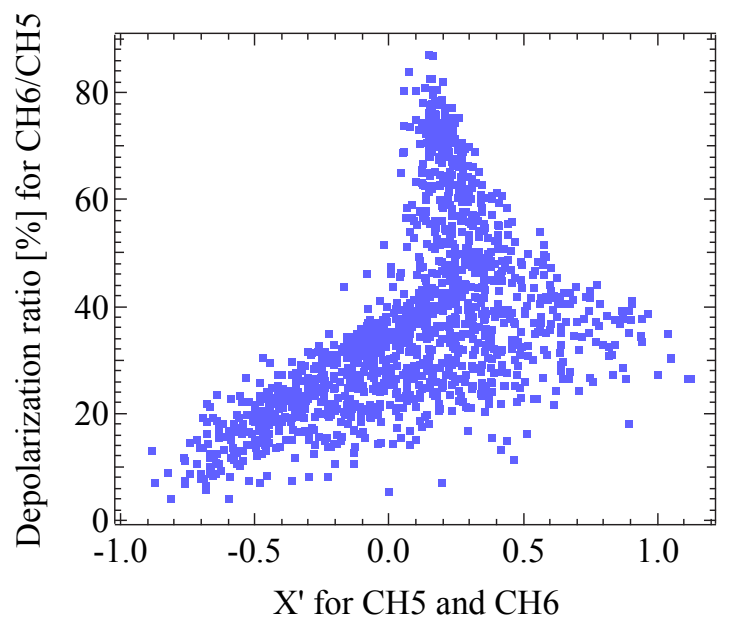

Figure 3 Relationship between the depolarization ratio and $X^{\prime}$ for $\mathrm{CH} 5$ and $\mathrm{CH} 6$ for the same clouds in Figure 1 .

\section{CONCLUSIONS}

The MFMSPL data were first used to examine and develop the cloud mask algorithms. The method 
was also extended to the algorithm for space-borne lidar. The MFMSPL data is especially suitable for the development of the algorithms that were applied to space-borne lidar where multiple scattering often plays a fundamental role.

It was found that our former cloud mask scheme (KU-mask) tended to underestimate bottom part of clouds and new one can overcome this issue.

The relation between depolarization ratio and $\mathrm{X}$ ' was analyzed by using the MFMSPL data for optically thick water clouds. Similar but somewhat different feature was found for off-beam channels compared with the diagram used in KU-type for the discrimination of cloud particle type. It was suggested that additional treatment might be needed when the discrimination of cloud particle type is conducted in the deeper part of the clouds where multiple scattering contributions are dominant.

\section{ACKNOWLEDGEMENTS}

CALIPSO data were obtained from the Atmospheric Sciences Data Center at NASA's Langley Research Center. This study was supported by JSPS KAKENHI Grant Numbers JP17H06139 and JP15K17762, by Green Network of Excellence (GRENE) Program Arctic Climate Change Project and the Arctic Challenge for Sustainability (ArCS), by the Japan Aerospace Exploration Agency for EarthCARE Research Announcement and by the Collaborative Research Program of Research Institute for Applied Mechanics, Kyushu University.

\section{References}

[1] Bissonnette, L. R., G. Roy, L. Poutier, S. G. Cober, and G. A. Isaac 2002: Multiplescattering lidar retrieval method: tests on Monte Carlo simulations and comparisons with in situ measurements, Appl. Opt. 41(30), 6307-6324

[2] Hu, Y., 2007: Depolarization ratio-effective lidar ratio relation: Theoretical basis for space lidar cloud phase discrimination, Geophys. Res. Lett. 34(11), L11812
[3] Yoshida, R., H. Okamoto, Y. Hagihara, and H. Ishimoto 2010: Global analysis of cloud phase and ice crystal orientation from CloudAerosol Lidar and Infrared Pathfinder Satellite Observation (CALIPSO) data using attenuated backscattering and depolarization ratio," J. Geophys. Res. 115, D00H32

[4] Okamoto, H., K. Sato, T. Nishizawa, N. Sugimoto, T. Makino, Y. Jin, and A. Shimizu, T. Takano, M. Fujikawa, 2016: Development of a multiple-field-of-view multiple-scattering polarization lidar: comparison with cloud radar, Opt. Express, 24(26), 30053-30067, https:// doi.org/10.1364/OE.24.030053

[5] Illingworth A., 2015: THE EARTHCARE SATELLITE: THE NEXT STEP FORWARD IN GLOBAL MEASUREMENTS OF CLOUDS, AEROSOLS, PRECIPITATION AND RADIATION, Bull. Amer. Meteor. Soc., 96, 1311-1332, doi: http://dx.doi.org/10.1175/ BAMS-D-12-00227.1

[6] Hagihara, Y., H. Okamoto, and R. Yoshida 2010: Development of combined CloudSat/CALIPSO cloud mask to show global cloud distribution, 2010, J. Geophys. Res., 115, D00H33, doi:10.1029/ 2009JD012344

[7] Okamoto, H., Nishizawa T., Takemura, T., Kumagai, H., Kuroiwa, H., Sugimoto, N., Matsui, I., Shimizu, A., Emori, S., Kamei, A., Nakajima, T., 2007: Vertical cloud structure observed from shipborne radar and lidar: Midlatitude case study during the MR01/K02 cruise of the research vessel Mirai, J. Geophys. Res. Atmos. 112, D08216, doi:10.1029/2006JD007628.

[8] Okamoto, H., Nishizawa, T., Takemura, T., Sato, K., Kumagai, H., Ohno, Y., Sugimoto, N., Shimizu, A., Matsui, I., Nakajima, T., 2008, Vertical cloud properties in the tropical western Pacific Ocean: Validation of the CCSR/NIES/FRCGC GCM by shipborne radar and lidar, J. Geophys. Res. Atmos. 113, D24213, doi:10.1029/2008JD009812 